\title{
Fetal Diagnosis of Tetralogy of Fallot with Absent Pulmonary Valve Syndrome: Role of Fetal MRI
}

\author{
Osamauh Aldoss ${ }^{1}$, Yasuko Yamamura', James St.Louis', Tara Holm¹, Shanthie Sivanandam ${ }^{1}$ \\ ${ }^{1}$ Department of Pediatrics, Surgery, Obstetrics and Gynecology and Radiology, University of Minnesota, Minneapolis, USA.
}

\section{Abstract}

We describe the beneficial contribution of fetal MRI in evaluating the site and extent of airway compression by the dilated pulmonary arteries in a case of Tetralogy of Fallot absent pulmonary valve syndrome (TOFAPVS) diagnosed by fetal echocardiography at 20 weeks gestation age. Although, the fetal echocardiography is an adequate tool to establish the diagnosis of TOFAPVS which can be done accurately as early as 20 weeks gestation; fetal MRI should be considered as an adjuvant tool to evaluate the site and extent of tracheo-bronchial tree compression in patients with TOFAPVS.

Keywords: Congenital Heart Disease, Tetralogy of Fallot, Fetal MRI, Fetal echocardiography, Bronchial compression.

Corresponding Author: Dr.Shanthi Sivanandam MD, Division of Pediatric Cardiology, University of Minnesota, 2450 Riverside Ave, Minneapolis, Minnesota, 55454 USA.

Received: January 2018

Accepted: February 2018

\section{Introduction}

TOFAPVS is a rare variant of Tetralogy of Fallot (TOF). Incidence of TOFAPVS is 3-6\% of all infants with TOF and $0.2-0.4 \%$ of all infants with congenital heart disease (CHD). ${ }^{[1]}$ The perinatal mortality rate is high especially in the presence of ductus arteriosus. ${ }^{[2]}$ Agenesis of the ductus arteriosus is a frequent association of this syndrome. Miller et al. was the first to report a case with TOFAPVS in 1962. ${ }^{[3]}$ Conventional fetal echocardiography including 2D, color and pulse Doppler tool is usually adequate to make the diagnosis of TOFAPVS. However, detection of this condition was described to be of a low yield before 21-22 weeks of gestation secondary to incomplete expression of the disease at that point. ${ }^{[2,4]}$ Our case was one of the few in the literature where diagnosis was made as early as 20 week GA by fetal echocardiography. Since bronchial compression is a major determinant in the outcome of these patients, careful evaluation of the bronchial tree is mandated. Mainstem bronchi are difficult to identify by the fetal ultrasound. For that reason, our patient underwent fetal MRI to evaluate the degree and extent of bronchial compression to facilitate planning for postnatal management. In our case, fetal MRI was clearly able to determine the size of main and the branch pulmonary arteries as well as the extent of bronchial obstruction. While fetal MRI has been used to evaluate fetal anatomy, particularly in cases of fetal anomalies, there are few reports in the literature reporting fetal MRI to assess fetuses with congenital heart disease. ${ }^{[5]}$ Up to our knowledge, we are the first to report the use of fetal MRI for bronchial tree evaluation in patient with TOFAPVS.

\section{Case Report}

A 25-year-old primigravida was referred forcomprehensive ultrasound and fetal echocardiography at 20 weeks gestation (GA). A detailed fetal echocardiogram demonstrated rudimentary pulmonary valve, cardiomegaly, significantly dilated main pulmonary artery [Main pulmonary artery 9 $\mathrm{mm}(\mathrm{z}$-score +6$)$, left pulmonary artery $8 \mathrm{~mm}(\mathrm{z}$-score +7.5$)$ and right pulmonary artery $9 \mathrm{~mm}(\mathrm{z}$-score +8$)$ ], severe pulmonary insufficiency, pulmonary stenosis with peak gradient of $25 \mathrm{mmHg}$, malalignment of large anterior ventricular septal defect with overriding of aorta and absent ductus arteriosus, findings consistent with TOFAPVS. The remainder of the fetal anatomy was unremarkable. Amniocentesis revealed a normal (46, XX) karytoype. Locus specific fluorescence in-situ hybridization revealed a deletion within the proximal long arm of chromosome 22 [del (22) (q11.2q11.2)]. A fetal MRI was performed at 31 week GA to evaluate the tracheo-bronchial tree, which demonstrated displacement and compression of the left main stem bronchus by the dilated left pulmonary arteries. The carina and right mainstem bronchus were also posteriorly displaced by the dilated pulmonary arteries. There was no increased $\mathrm{T} 2$ signal intensity or hemidiaphragm eversion to suggest trapping of fluid within the lung from bronchial obstruction. Comprehensive interdisciplinary services were 


\section{Aldos et al; Fetal Diagnasis of Tetralagy of Fallat with Alesent Pulmanary Value Syndrame}

involved at delivery and postnatal care. The patient underwent a primary cesarean section at 39 weeks without complications. A 2820 grams female infant was delivered with APGAR scores of 6,6 , and 8 at 1,5 and 10 minutes, respectively. The infant was found to be cyanotic with poor respiratory effort. The infant was intubated and mechanically ventilated immediately after birth. Post natal echocardiogram confirmed the diagnosis of TOFAPVS with moderate pulmonary valve stenosis. The Main pulmonary artery (MPA) diameter measured $19 \mathrm{~mm}(\mathrm{z}$-score +5.9) and left pulmonary artery (LPA) and right pulmonary artery (RPA) measured $16 \mathrm{~mm}$ (z-score +8). The infant had difficult ventilation and oxygenation on mechanical ventilation in the first day of life even with nitric oxide use. A trial of prone position showed dramatic improvement in the oxygenation and ventilation. A bronchoscopy was performed on day 3 of life and showed significant compression of both mainstem bronchi, confirming the fetal MRI findings. At one week of age, the infant underwent a complete one-stage surgical repair which included VSD closure, right ventricular outflow tract muscle bundle resection, trans- annular patch, reduction pulmonary arterioplasty and leftpulmonary arteriopexy. The infant needed inotropic support for few days after the surgery and she was successfully extubated on post-operative day 5 . Post-operative echocardiogram showed no residual shunts and mild residual pulmonary stenosis. Pulmonary arteries sizes were significantly smaller [MPA $7 \mathrm{~mm}$ (z-score +0.9$)$, RPA $7 \mathrm{~mm}(\mathrm{z}$-score +2.5), and LPA $6 \mathrm{~mm}(\mathrm{z}$-score +1.9)].

\section{Discussion}

TOFAPVS is a rare variant of TOF. Although the anatomic features are similar to classic TOF, the physiology and disease progression is significantly different. TOF was first described by Fallot in 1888 and includes pulmonary outflow obstruction, ventricular septal defect, overriding aorta and right ventricular hypertrophy. ${ }^{[6]}$ These structural changes are a sequence of infundibular distortion related to anterior malalignment of the conal ventricular septum. Common findings of TOFAPVS are cardiomegaly, right ventricular outflow view showing dysplastic pulmonary valve leaflets and different degrees of dilatation of the main and branch pulmonary arteries. ${ }^{[7]}$

The presence of rudimentary pulmonary valve or complete absence of the valve leads to significant fetal pulmonary regurgitation. The chronic right ventricular overload is transmitted to the pulmonary arteries and leading to dilatation of these vessels with subsequent compression of the tracheobronchial tree. Color flow imaging will show significant pulmonary insufficiency and pulse Doppler shows varying degree of pulmonary stenosis; although, detection of this condition was described to be of a low yield before 21-22 weeks secondary to incomplete expression of the disease at that point. ${ }^{[8]}$

Since bronchial compression is a major determinant in the outcome of these patients, careful evaluation of the bronchial tree is mandated. Mainstem bronchi are difficult to identify by fetal ultrasound. Therefore, fetal MRI can prove to be a useful tool to evaluate the degree and extent of bronchial compression and to facilitate the planning for postnatal management.

Mortality and morbidity rates in patients with TOFAPVS far exceed those patients with typical TOF. Perinatal mortality is still high despite the ability for early prenatal diagnosis. ${ }^{[2]}$ Patients are at risk for hypoxemia, heart failure, respiratory failure, or a combination of these factors. However, bronchial compression is believed to be the major determinant of mortality and morbidity. Fetuses with TOFAPVS should be delivered in a perinatal center having neonatal and cardiac intensive care units and experienced pediatric team. The combination of a reduction arterioplasty and pulmonary arteriopexy appears to make more space in the mediastinum and lifts the pulmonary arteries off the tracheobronchial tree. Rabinovitch et al described abnormal tufts of smaller pulmonary arteries that compress the intrapulmonary bronchi as well as a decrease in the number of alveoli in patients with TOFAPVS. These findings might explain why surgical relief of main airway obstruction alone is not always effective in reversing the obstructive airway disease. ${ }^{[7]}$

In our case, fetal MRI was clearly able to determine the size of main and the branch pulmonary arteries as well as the extent of bronchial obstruction. While fetal MRI has been used to evaluate fetal anatomy, particularly in cases of fetal anomalies, there are very few reports in the literature reporting fetal MRI to assess fetuses with congenital heart disease. Taragin et al reported 2 cases of TOFAPVS who underwent postnatal chest MRI for movement continues to be a problem encountered with fetal MRI; evaluating bronchial compression postoperatively secondary to persistent respiratory insufficiency. ${ }^{[9]}$ Artifact associated with fetal nonetheless high quality images can be obtained by using very short acquisition time sequences, by using the current sequence as the scout for the next sequence, and by having an experienced MRI technologist or radiologist present.

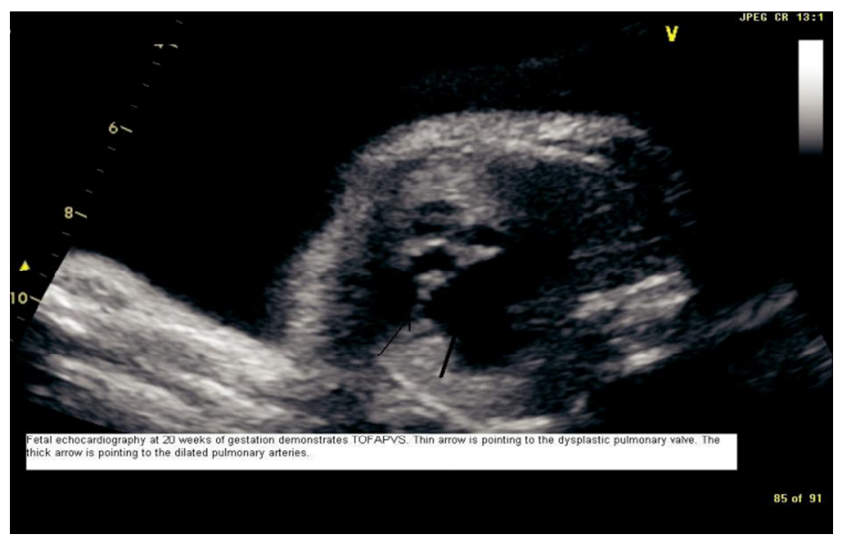




\section{Aldos et al; Fetal Diagnasis of Tetralagy of Fallat with Alesent Pulmanary Value Syndrame}

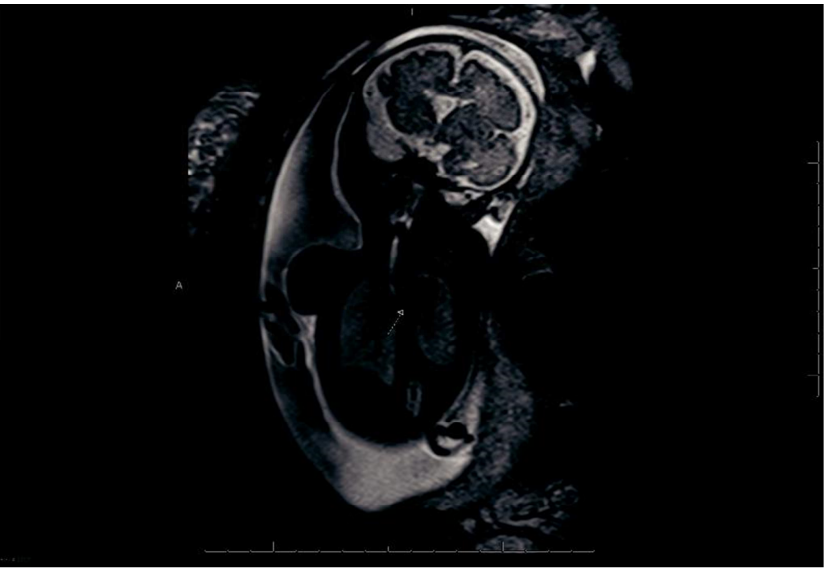

Figure 1 and 2: Fetal echocardiograph at 20 weeks of gestation demonstrates TOFAPVS .Thin arrow pointing to the dysplastic pulmonary valve. The thick arrow is pointing to the dilated pulmonary arteries

\section{Conclusion}

We conclude that fetal echocardiography is an adequate tool for TOFAPVS diagnosis and that the diagnosis can be made as early at 20 weeks gestation; however fetal MRI should be considered an adjuvant tool to evaluate the site and extent of tracheo-bronchial tree compression in these patients. We strongly recommend the use of fetal MRI in patients with prenatal diagnosis of TOFAPVS. Comprehensive interdisciplinary services are required to facilitate management of these high risks patients.

\section{References}

1. Volpe P, Paladini D, Marasini M, Buonadonna AL, Russo MG, Caruso G, et al. Characteristics, associations and outcome of absent pulmonary valve syndrome in the fetus. Ultrasound Obstet Gynecol 2004;24(6):623-628.

2. Galindo A, Gutierrez-Larraya F, Martinez JM, Del Rio M, Graneras A, Velasco JM, et al. Prenatal diagnosis and outcome for fetuses with congenital absence of the pulmonary valve. Ultrasound Obstet Gynecol 2006;28(1):32-39.

3. Miller RA, Lev M, Paul MH. Congenital absence of the pulmonary valve. The clinical syndrome of tetralogy of Fallot with pulmonary regurgitation. Circulation 1962;26:266-278.

4. Becker R, Schmitz L, Guschmann M, Wegner RD, Stiemer B, Entezami M. Prenatal diagnosis of familial absent pulmonary valve syndrome: case report and review of the literature. Ultrasound Obstet Gynecol 2001;17(3):263-267.

5. Recio Rodriguez M, Martinez de Vega V, Cano Alonso, Perez Pedregosa J et al. MR imaging of thoracic abnormalities in the fetus. Radiographics 2012;32(7): E305-21

6. Neill CA and Clark EB. Tetralogy of Fallot. The first 300 years. Tex Heart Inst J 1994;21(4):272-279.

7. Rabinovitch M, Grady S, David I, Van Praagh R, Sauer U, Buhlmeyer $\mathrm{K}$, et al. Compression of intrapulmonary bronchi by abnormally branching pulmonary arteries associated with absent pulmonary valves. Am J Cardiol 1982; 50(4):804-813.

8. Sleurs E, De Catte L, Benatar A. Prenatal diagnosis of absent pulmonary valve syndrome in association with 22q11 deletion. J Ultrasound 2004;23(3):417-22.

9. Taragin BH, Berdon WE, Printz B. MRI assessment of bronchial compression in absent pulmonary valve syndrome and review of the syndrome. Pediatr Radiol 2006;36(1):71-75.

Copyright: () the author(s), publisher. Asian Journal of Clinical Pediatrics and Neonatology is an Official Publication of "Socicty for Health Care \& Research Development". It is an open-access article distributed under the terms of the Creative Commons Attribution NonCommercial License, which permits unrestricted non-commercial use, distribution, and reproduction in any medium, provided the original work is properly cited.

How to cite this article: Aldoss O, Yamamura Y, Louis JS, Holm T, Sivanandam S. Fetal Diagnosis of Tetralogy of Fallot with Absent Pulmonary Valve Syndrome: Role of Fetal MRI. Asian J. Clin. Pediatr. Neonatol.2018;6(1):3-5.

DOI: dx.doi.org/10.21276/ajcpn.2018.6.1.2

Source of Support: Nil, Conflict of Interest: None declared 